\section{Solid-phase Micro-Extraction}

C. Vidal ${ }^{1}$ und W.-R. Külpmann ${ }^{2}$

${ }^{1}$ Landeskriminalamt Niedersachsen, Dezernat 53 „Chemie“, Hannover, Deutschland

${ }^{2}$ Hannover, Deutschland

Synonym(e) Festphasen-Mikroextraktion; SPME

Englischer Begriff solid phase micro-extraction

Definition Verfahren der Probenvorbereitung für gaschromatographische Analysen ( $\triangleright$ Gaschromatographie).

Beschreibung Bei der SPME handelt es sich um ein Extraktionsverfahren, bei dem eine dünne Faser, die mit speziellen Adsorbenzien belegt ist, Analytmoleküle aus einer Matrix aufnimmt. Anschließend wird die Faser über eine Kanüle in das Einlasssystem eines Gaschromatographen verbracht und die Analyse durchgeführt. Es gibt verschiedene Verfahrensweisen für die SPME. Die Faser kann direkt in eine zu analysierende Flüssigkeit eingetaucht werden. Beim Vorliegen flüchtiger Analyten kann die Extraktion auch aus dem Dampfraum (Headspace) über einer zu analysierenden Flüssigkeit oder einer festen Probe (z. B. eingedampfter Extrakt) geschehen. Bei letzterer wird ein gasdichtes Gefäß mit der Probe erhitzt. Über die Kanüle einer Spritze wird die SPMEFaser in den Dampfraum des Probengefäßes eingebracht, sodass flüchtige Verbindungen aufgenommen werden können. S. a. > Festphasenextraktion, \ Mikrosäulen.

\section{Literatur}

Pawliszyn J (1997) Solid phase microextraction. Wiley-VCH, New York 